\title{
Operative Technique for En Bloc Resection of Upper Cervical Chordomas: Extended Transoral Transmandibular Approach and Multilevel Reconstruction
}

\author{
Luis Alberto Ortega-Porcayo ${ }^{1}$, Eibar Ernesto Cabrera-Aldana ${ }^{1}$, Nicasio Arriada-Mendicoa ${ }^{1}$, \\ Juan Luis Gómez-Amador ${ }^{1}$, Martín Granados-García ${ }^{2}$ Juan Barges-Coll ${ }^{1}$ \\ ${ }^{1}$ Department of Neurosurgery, National Institute of Neurology and Neurosurgery Manuel Velasco Suárez, Mexico \\ ${ }^{2}$ Department of Head and Neck Tumors, National Cancer Institute of Mexico, Mexico
}

Anterior exposure for cervical chordomas remains challenging because of the anatomical complexities and the restoration of the dimensional balance of the atlanto-axial region. In this report, we describe and analyze the transmandibular transoral approach and multilevel spinal reconstruction for upper cervical chordomas. We report two cases of cervical chordomas (C2 and C2-C4) that were treated by marginal en bloc resection with a transmandibular approach and anterior-posterior multilevel spinal reconstruction/fixation. Both patients showed clinical improvement. Postoperative imaging was negative for any residual tumor and revealed adequate reconstruction and stabilization. Marginal resection requires more extensive exposure to allow the surgeon access to the entire pathology, as an inadequate tumor margin is the main factor that negatively affects the prognosis. Anterior and posterior reconstruction provides a rigid reconstruction that protects the medulla and decreases axial pain by properly stabilizing the cervical spine.

Keywords: Transmandibular approach; Chordoma; Neurosurgical instrumentation; Spinal neoplasms

\section{Introduction}

Primary tumors of the spine comprise less than $10 \%$ of all spinal tumors $[1,2]$. The cervical spine is the most commonly involved mobile segment [1]. The course of the disease is slow, and death can result from complications related to local extension [3].

En bloc resection with tumor-free margins appears to be the only treatment that results in a continuous diseasefree course, and this treatment provides superior local control compared to intralesional incomplete excision and radiation therapy [4]. Anterior exposure for cervical chordomas remains challenging because of the anatomical complexities and restoration of the dimensional balance of the atlanto-axial region.

\section{Technical Note}

\section{Clinical findings}

1) First case

A 43-year-old man presented a 3-month history of dif-

Received Mar 16, 2014; Revised Apr 25, 2014; Accepted Apr 25, 2014

Corresponding author: Juan Barges-Coll

Division of Neurological Surgery, National Institute of Neurology and Neurosurgery Manuel Velasco Suárez,

3877 Insurgentes Sur, Tlalpan, Mexico City ZC 14269, Mexico

Tel: +5255-5606-3822, Fax: +5255-5673-6432, E-mail: jbargescoll@mac.com

*Contents of this work were presented in poster form at the 35 Neurosurgical Latin-American Congress, Rio de Janeiro, Brazil, April 4, 2012. 
fuse, dull cervical pain with paresthesia in the arms and legs. The patient had incomplete sensory and motor impairments. Computed tomography (CT) and magnetic resonance imaging (MRI) revealed a heterogeneous midline lytic vertebral lesion extending from $\mathrm{C} 2$ to $\mathrm{C} 4$ with retropharyngeal and paravertebral extensions (Fig. 1AC). The tumor had epidural extension and resulted in anterior medullar compression with right vertebral artery encasing (Fig. 1D-F).

\section{2) Second case}

A 23-year-old woman presented an 8-month history of dull cervical pain associated with paresthesia in both arms. One month prior to admission, she started experiencing decreased muscle strength in both legs and subsequently in both arms. CT and MRI revealed a heterogeneous, midline lytic vertebral lesion, extending from $\mathrm{C} 2$ to $\mathrm{C} 3$. The tumor extended ventrally to the anterior longitudinal ligament with epidural extension dorsally and anterior medullar compression (Fig. 2A).

\section{3) Staging}

The American Spinal Injury Classification Impairment
Scale [5] and Frankel scale were D in both cases. Both patients were diagnosed with a tomography-guided Trucut needle biopsy (Fig. 2D). First case: Enneking et al. [6] IB and Weinstein-Boriani-Biagini (WBB) classification [7] concentric extension A-F and radial extension 3-9. Second case: Enneking 1B and WBB A-E/5-8.

\section{Surgical technique}

\section{1) First case}

The patient was placed in the prone position. We used a midline incision from inion to $\mathrm{C} 7$ with subsequent midline posterior cervical muscles dissection. Posterior bilateral laminectomies and facetectomies of $\mathrm{C} 2-\mathrm{C} 4$ were performed. The left vertebral artery was dissected away from the tumor margin. The right vertebral artery was encased $270^{\circ}$ by the tumor, the distal portion of the vertebral artery that was tumor-free was clamped with a nontraumatic vascular clamp and somatosensory-evoked potentials (SSEP) were recorded during the next $30 \mathrm{~min}$ utes. No changes were detected, and a second proximal clip was used to resect the vertebral artery along with the tumor. The $\mathrm{C} 2-\mathrm{C} 4$ nerve roots on the right side were also
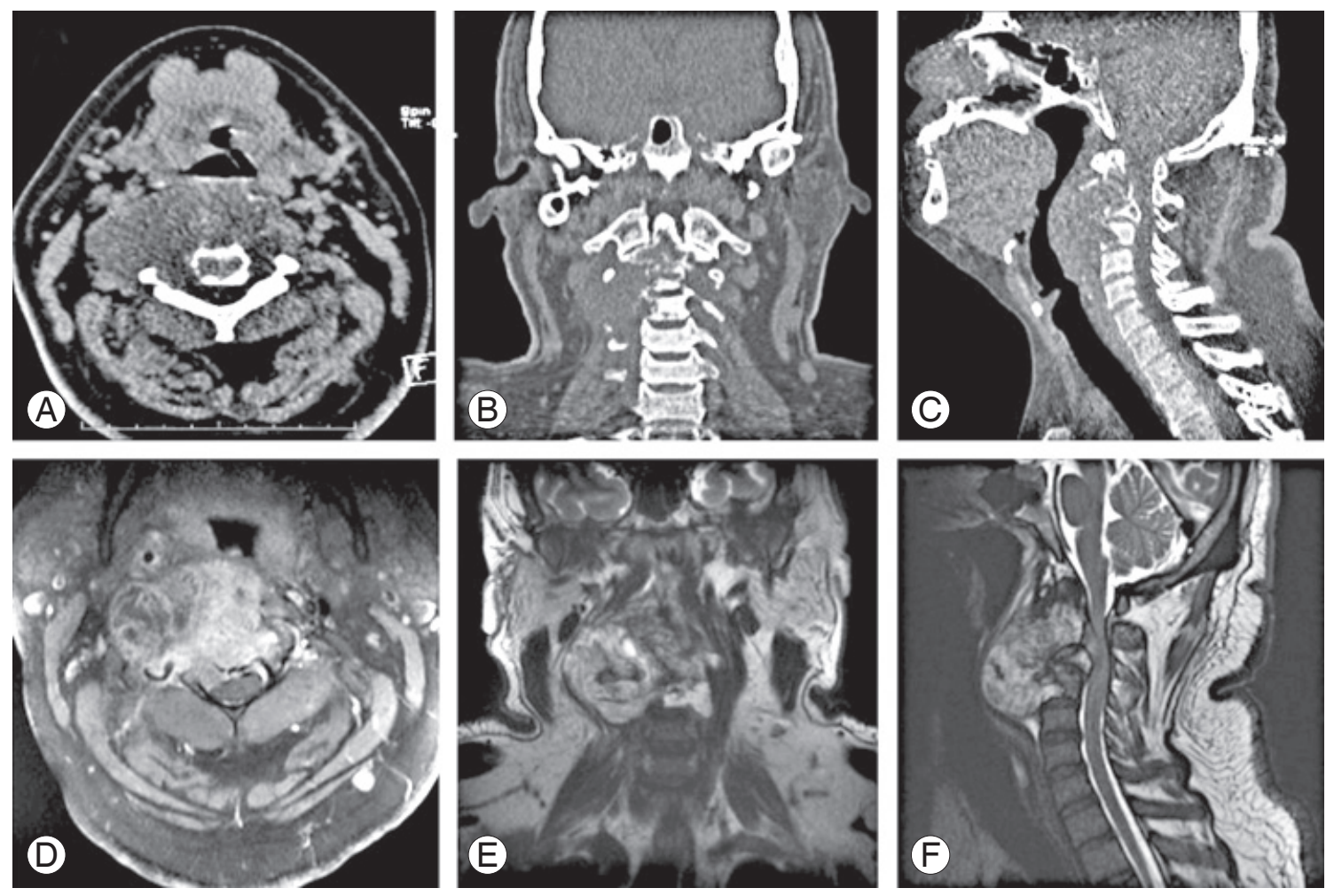

Fig. 1. Preoperative images of the first case. Computed tomography (A, axial; $\mathbf{B}$, coronal; and $\mathbf{C}$, sagittal) and magnetic resonance imaging (D, axial; E, coronal; and F, sagittal). 
encased and transected. Occipito-cervical fixation was performed using contoured titanium rods fixated with four titanium screws for occipital fixation and six polyaxial titanium screws for lateral mass at C5-C7 (Fig. 3). The posterior bony elements were completely removed to facilitate en bloc spondylectomy at the second stage of the procedure.

Second stage: The patient was placed in the supine position (Fig. 4). A tracheostomy tube was placed, and a midline skin incision from the lower lip to the hyoid bone was made. The mandible and the hyoid bone were exposed, and a midline mandibulotomy was performed using a reciprocating saw. A midline glossotomy allowed direct observation of the posterior pharyngeal wall. The soft palate, pharyngeal mucosa, constrictor muscles, buccopharyngeal fascia and prevertebral muscles were split and retracted. The clivus, cranio-cervical junction, and $\mathrm{C} 1-\mathrm{C} 4$ were exposed. Peripheral devascularization of the retropharyngeal and paravertebral extension of the tumor was performed with bipolar coagulation. Discectomy of C4-C5 allowed visualization of the ventral dura, which was not infiltrated and was preserved, providing us the dorsal limit of our resection (Fig. 5A). After resecting the posterior longitudinal ligament, spondylectomy of $\mathrm{C} 2-$ $\mathrm{C} 4$ was performed by en bloc drilling of the inferior and superior borders with a diamond drill at the transverse foramen. After the en bloc marginal resection (Fig. 5B),

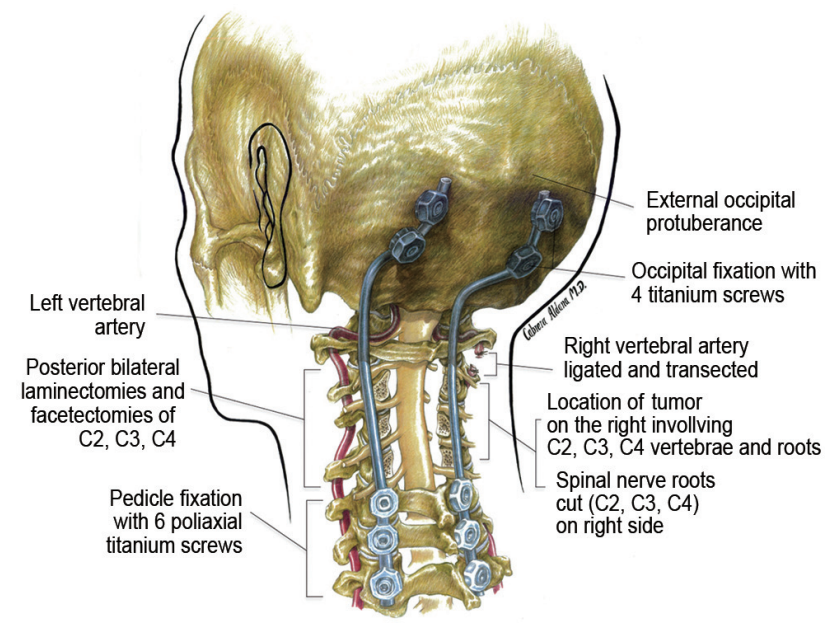

Fig. 3. Artist's illustration of the posterior stage (occipitocervical fixation) of the surgery from the first case.
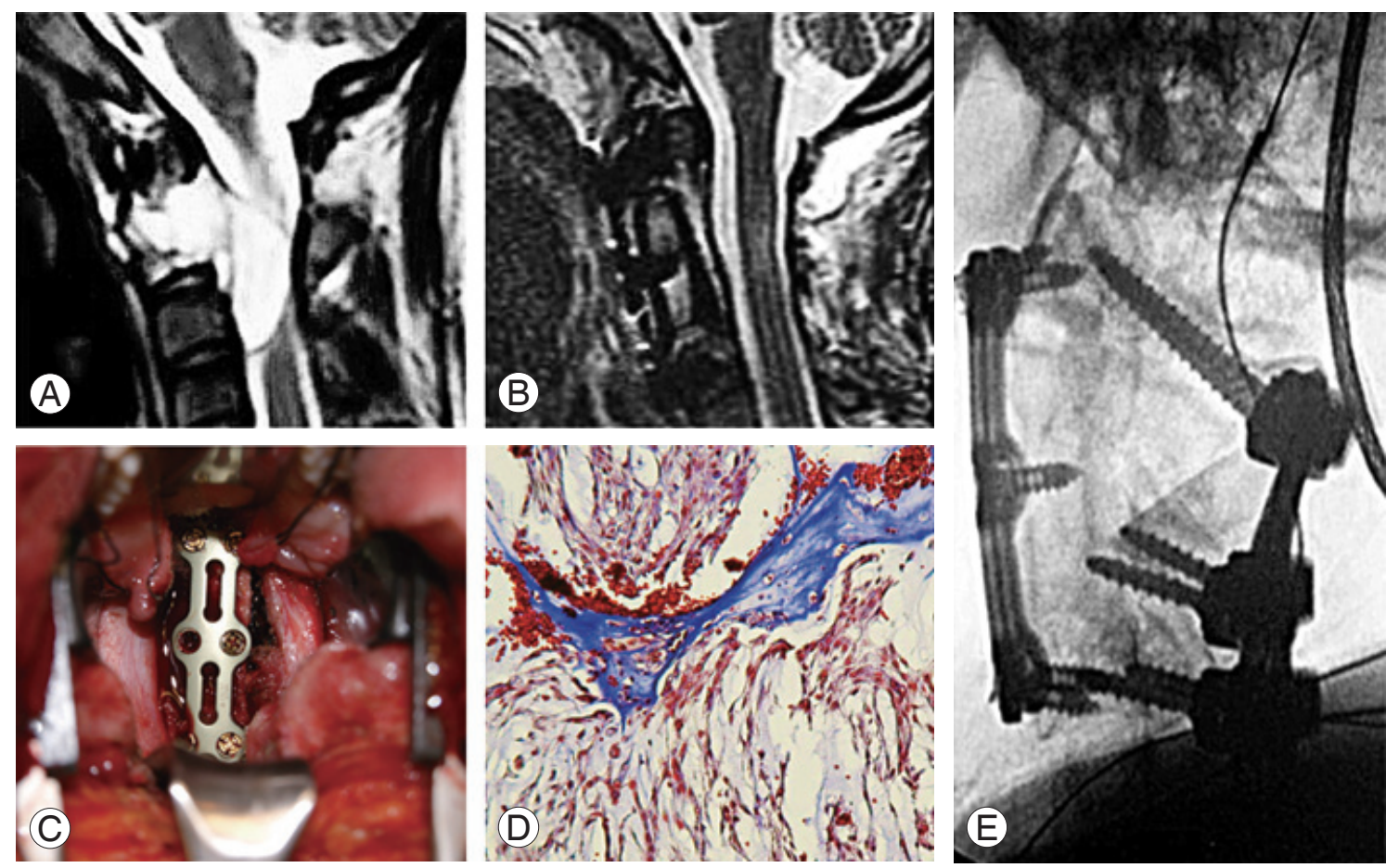

Fig. 2. Second case. (A) Preoperative sagittal magnetic resonance imaging (MRI). (B) Postoperative sagittal MRI reveals no residual tumor. (C) Intraoperative photograph, showing the anterior cervical plate placed from C1-C4. (D) Microscopic photograph, demonstrating classical tumor lobules separated by bands of fibrous tissue (Masson's trichrome stain, $\times 50$ ). (E) Lateral plain radiograph of the two-column reconstruction. 
reconstruction and anterior fixation was completed with a titanium cage filled with bone matrix and fixated with an anterior plate with two bicortical $\mathrm{C} 1$ and $\mathrm{C} 5$ screws

(Figs. 5C, 6D). The pharyngeal wall, constrictor prever-

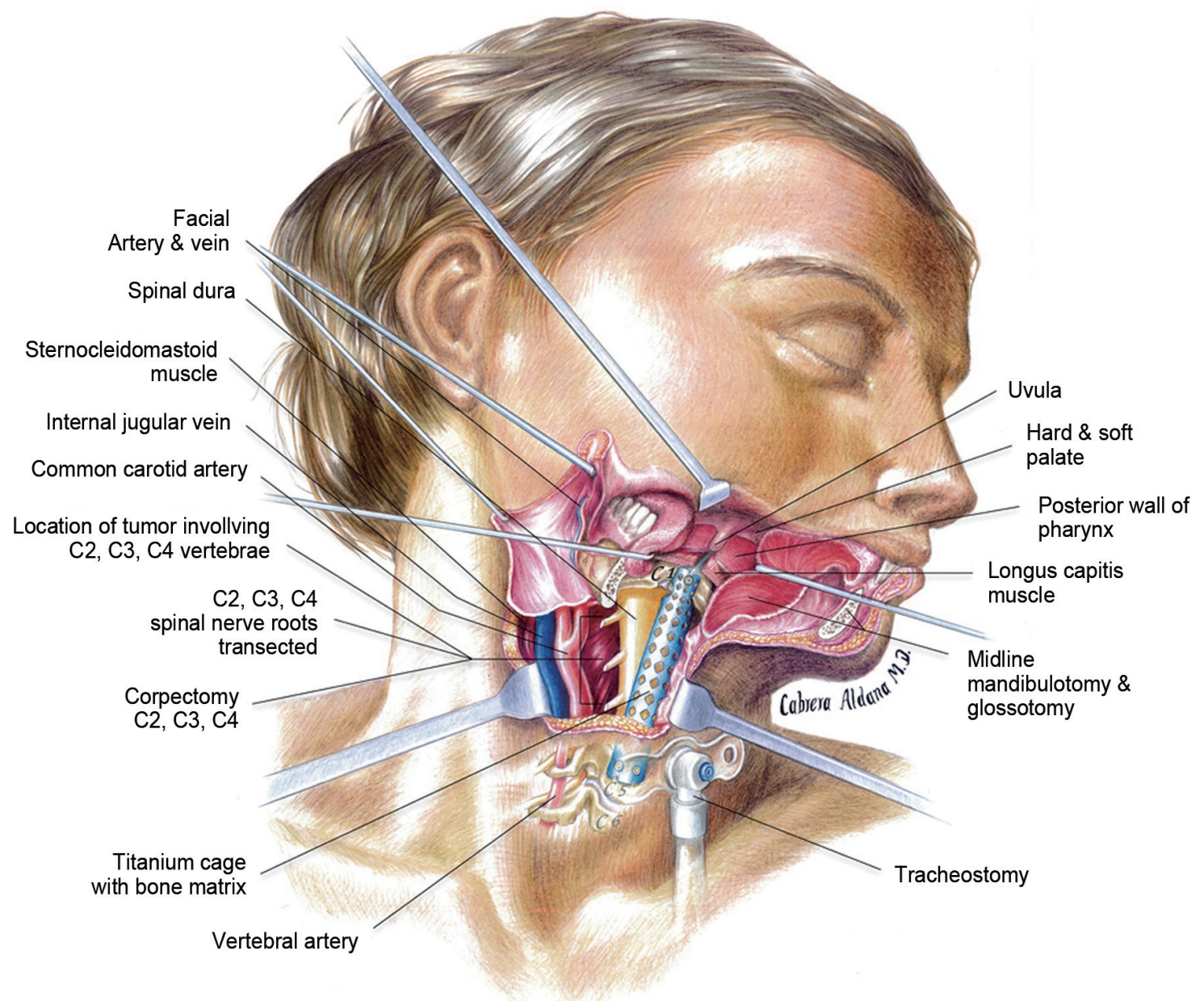

Fig. 4. Artist's illustration of the anterior stage of the surgery from the first case. Extended oral transmandibular-transpharyngeal approach. Notice the extension of the approach with the addition of a glossotomy and mandibular osteotomy. The illustration shows the titanium cage before the anterior plate fixation.
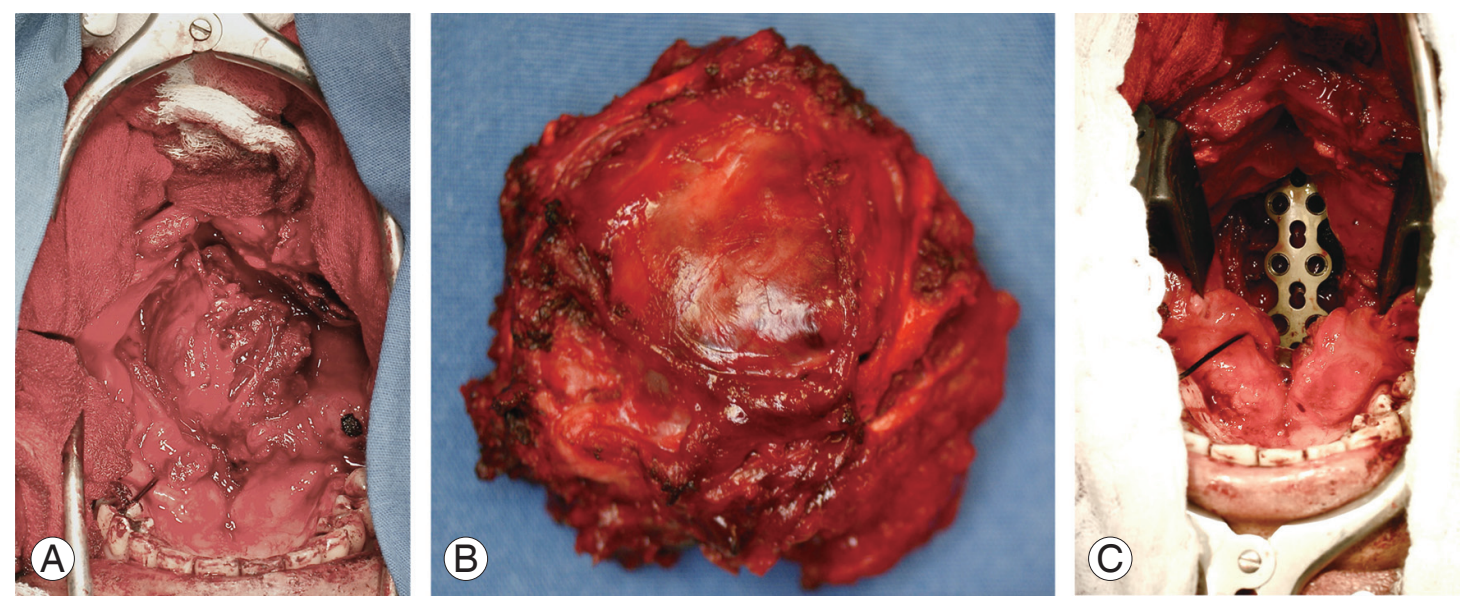

Fig. 5. Intraoperative images. (A) Notice the wide view allowed by the approach favoring marginal resection. (B) En bloc specimen. (C) Anterior reconstruction with a titanium cage filled with bone matrix and fixated with a plate. 
tebral muscles, soft palate, and the tongue were approximated with interrupted 2-0 Vicryl. The mandible was approximated and secured with two plates that were fixated with four screws. Midline muscles were approximated with interrupted 2-0 Vicryl and the skin was closed with interrupted 3-0 nylon.

\section{2) Second case}

First stage: Both vertebral arteries were isolated and tumor invasion was not visualized. Cervical rod fixation was performed with lateral mass screws $\mathrm{C} 1, \mathrm{C} 3$, and $\mathrm{C} 4$ (Fig. 2E).

Second stage: C3-C4 discectomy and corpectomies of
C2 and C3 were performed. C2-C3 nerve roots from the left side were transected. A high-speed drill was used to cut across the base of the dens. This established the superior margin of the resection. No dura mater invasion was observed. The reconstruction and anterior fixation were carried out with a non-vascularized autologous fibular graft that was cut to size and fashioned to form a sharp upper end, which was embedded into the residual dens. The inferior end of the graft rested against the superior endplate of $\mathrm{C} 4$. An anterior cervical plate was then placed from $\mathrm{C} 1$ to $\mathrm{C} 4$, with two screws penetrating the anterior arch of $\mathrm{C} 1$, two penetrating $\mathrm{C} 4$, and one penetrating the fibular graft (Fig. 2C).
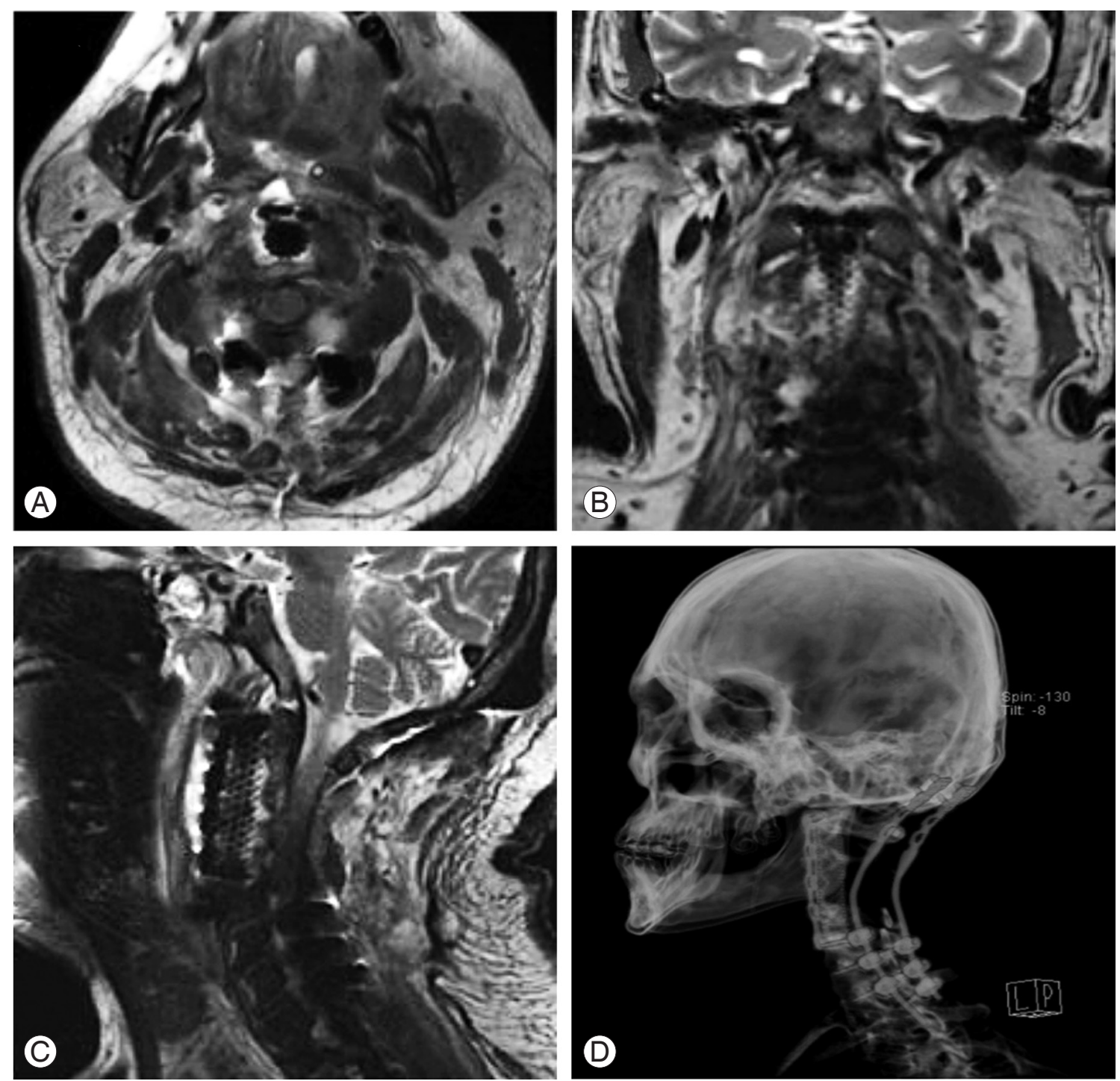

Fig. 6. Postoperative images of the first case. Magnetic resonance imaging (A, axial; B, coronal; and $\mathbf{C}$, sagittal) reveals no evidence of residual tumor and (D) the postoperative three-dimensional sagittal computed tomography reconstruction. 


\section{Postoperative course}

Surgical times were 9 hours and 5 hours, respectively for both procedures with a blood loss of 2,100 $\mathrm{mL}$ for the first patient and $900 \mathrm{~mL}$ for the second patient. Both patients required nasogastric-feeding tubes that were removed on postoperative day 5 , and the patients were started on fluids. Sialorrhea was observed for 1 week. A rigid collar was used for 2 months. The exteroceptive sensory and motor functions improved to active movement and full range of motion against full resistance, with postoperative ASIA E and Frankel E scores. They continued aggressive rehabilitation, and postoperative imaging was negative for any residual tumor and revealed adequate reconstruction and stabilization of the spine (Figs. 2B, E, 6). The patients have now been followed up for 2 years without any hardware failure. Patient 2 has completely fused. Patient 1 does not have fusion of posterior elements but remains without signs of hardware failure.

\section{Discussion}

\section{Approach}

Marginal resection requires a more extensive exposure to allow the surgeon access to the entire pathology [8]. Cervical anterolateral approaches, such as the retrocarotid lateral [9] or anterior retropharyngeal approaches [10] have been proposed to decrease significant morbidity; however, degree of exposure attained with these lateral approaches may be limited for tumors such as chordomas. We favor the extended transoral approach (Fig. 4), which provided us direct access to C3-C4 caudally and a wider surgical field. The midline allows the surgeon greater exposure without severing major muscles, important blood vessels, or nerves. Glossotomy is necessary when the lesion extends down to C2 or below [11]. As described previously by Arbit and Patterson [12], the cosmetic deformity and functional loss are minimal despite the seemingly radical incision.

The limitations encountered for cervical spine chordomas result from involvement of the dura, nerve roots, and vertebral arteries $[3,9]$. In our cases the duramater was preserved, but the resection included the right $\mathrm{C} 2-4$ roots and right vertebral artery in one case (Fig. 3 ) and the $\mathrm{C} 2-3$ left roots in the other case with minimal functional compromise; only mild dyspnea was documented for 3 days. Although there are few reports of serious complications, the risk is significant despite variability in the radiculomedullary branches [3]. When a vertebral artery is involved, it has to be dissected above and beneath the encasing before attempting for marginal resection. An intraoperative occlusion test of the vertebral artery with SSEP is highly recommended to avoid fatal complications.

\section{Reconstruction}

Anterior and posterior reconstructions are necessary after en bloc resection [13]. Instability after C2-C4 corpectomies was corrected by adding a single anterior cage that was screwed with an anterior plate to the remaining osseous structures (Fig. 6D). In the second case, we used an autologous fibula graft and a cervical plate instead of the cage. Both were useful for the anterior column restoration. We will compare the rates of fusion over time.

The decision to perform occipitocervicothoracic fusion is difficult because of the functional limitations it places on the patient by greatly reducing spinal mobility [14]. We decided to perform an occipito-cervical fixation in the first patient (Fig. 3) as we believed that stabilization of three segments below our resection was sufficient to provide biomechanical support with less functional limitation. In the second patient, the risk of construct failure was lower because it was a shorter segment $(\mathrm{C} 2-\mathrm{C} 3)$; therefore, we decided to perform cervical fixation (Fig. 2E).

En bloc resection is feasible in the cervical spine and may offer potential for a cure, particularly because the role of adjuvant therapy in chordomas of the cervical spine is not well defined. Even for large tumors that involve a vertebral artery as in case 1 , the decision to occlude the vessel should be done using angiography or SSEP. Anterior and posterior reconstruction is extremely important, as it provides a rigid reconstruction that protects the medulla and decreases axial pain by properly stabilizing the cervical spine.

\section{Conflict of Interest}

No potential conflict of interest relevant to this article was reported.

\section{References}

1. Ropper AE, Cahill KS, Hanna JW, McCarthy EF, 
Gokaslan ZL, Chi JH. Primary vertebral tumors: a review of epidemiologic, histological and imaging findings, part II: locally aggressive and malignant tumors. Neurosurgery 2012;70:211-9.

2. McMaster ML, Goldstein AM, Bromley CM, Ishibe N, Parry DM. Chordoma: incidence and survival patterns in the United States, 1973-1995. Cancer Causes Control 2001;12:1-11.

3. Rhines LD, Fourney DR, Siadati A, Suk I, Gokaslan ZL. En bloc resection of multilevel cervical chordoma with C-2 involvement. Case report and description of operative technique. J Neurosurg Spine 2005;2:199205.

4. Boriani S, Bandiera S, Biagini R, et al. Chordoma of the mobile spine: fifty years of experience. Spine (Phila Pa 1976) 2006;31:493-503.

5. Maynard FM Jr, Bracken MB, Creasey G, et al. International Standards for Neurological and Functional Classification of Spinal Cord Injury. American Spinal Injury Association. Spinal Cord 1997;35:266-74.

6. Enneking WF, Spanier SS, Goodman MA. A system for the surgical staging of musculoskeletal sarcoma. 1980. Clin Orthop Relat Res 2003;(415):4-18.

7. Boriani S, Weinstein JN, Biagini R. Primary bone tumors of the spine. Terminology and surgical staging. Spine (Phila Pa 1976) 1997;22:1036-44.
8. Hsu W, Wolinsky JP, Gokaslan ZL, Sciubba DM. Transoral approaches to the cervical spine. Neurosurgery 2010;66(3 Suppl):119-25.

9. Barrenechea IJ, Perin NI, Triana A, Lesser J, Costantino P, Sen C. Surgical management of chordomas of the cervical spine. J Neurosurg Spine 2007;6:398-406.

10. Yang X, Wu Z, Xiao J, et al. Sequentially staged resection and 2-column reconstruction for $\mathrm{C} 2$ tumors through a combined anterior retropharyngealposterior approach: surgical technique and results in 11 patients. Neurosurgery 2011;69(2 Suppl Operative):ons184-93.

11. Delgado TE, Garrido E, Harwick RD. Labiomandibular, transoral approach to chordomas in the clivus and upper cervical spine. Neurosurgery 1981;8:6759.

12. Arbit E, Patterson RH Jr. Combined transoral and median labiomandibular glossotomy approach to the upper cervical spine. Neurosurgery 1981;8:672-4.

13. Cloyd JM, Chou D, Deviren V, Ames CP. En bloc resection of primary tumors of the cervical spine: report of two cases and systematic review of the literature. Spine J 2009;9:928-35.

14. Jackson RJ, Gokaslan ZL. Occipitocervicothoracic fixation for spinal instability in patients with neoplastic processes. J Neurosurg 1999;91(1 Suppl):81-9. 